\title{
Near-BPS Skyrmions: Non-shell configurations and Coulomb effects
}

\author{
Eric Bonenfant, Louis Harbour and Luc Marleau \\ Département de Physique, de Génie Physique et d'Optique, \\ Université Laval, Québec, Québec, Canada G1K 7 P4
}

(Dated: September 1, 2018)

\begin{abstract}
The relatively small binding energy in nuclei suggests that they may be well represented by nearBPS Skyrmions since their mass is roughly proportional to the baryon number $A$. For that purpose, we propose a generalization of the Skyrme model with terms up to order six in derivatives of the pion fields and treat the nonlinear $\sigma$ and Skyrme terms as small perturbations. For our special choice of mass term (or potential) $V$, we obtain well-behaved analytical BPS-type solutions with nonshell configurations for the baryon density, as opposed to the more complex shell-like configurations found in most extensions of the Skyrme model. Along with static and (iso)rotational energies, we add to the mass of the nuclei the often neglected Coulomb energy and isospin breaking term. Fitting the four model parameters, we find a remarkable agreement for the binding energy per nucleon $B / A$ with respect to experimental data. These results support the idea that nuclei could be near-BPS Skyrmions.

PACS numbers: 12.39.Dc, 11.10.Lm
\end{abstract}

\section{INTRODUCTION}

The idea suggested by Skyrme [1] that baryon physics could emerge as solitons from an effective Lagrangian of meson fields remains one of the most original and successful attempts for the description of the low-energy regime of the theory of strong interactions (QCD). Although it predates QCD and was almost eclipsed by it, the proposal gained strong support when it was realized that, in the large $N_{c}$ limit, QCD is equivalent to an effective theory of mesons [2, 3]. Perhaps the most important feature of the Skyrme model in that regard is that the soliton solutions which arise are characterized by a conserved topological charge, the winding number, which Skyrme identified as the baryon number. In other words, in this scheme, the baryons as well as nuclei are simply topological solitons.

In its original formulation, the Skyrme model succeeds in predicting the properties of the nucleon within a precision of $30 \%$. This is considered a rather good agreement for a two-parameter theory [4]. However, a number of generalizations of the model have been proposed to improve this concordance with baryon and nuclear physics. They mostly exploit our ignorance of the exact form of the low-energy effective Lagrangian of QCD for example, the structure of the mass term [5 7], the contribution of other vector mesons [8, 9] or simply the addition of higher-order terms in derivatives of the pion fields [5]. Unfortunately, for now, QCD alone only gives hints that such extensions should appear and the complete determination of the effective Skyrme-like Lagrangian remains a most serious challenge.

Despite such efforts, one of the recurring problems of Skyrme-like Lagrangians is that they almost inevitably lead to large binding energy for nuclei already at the classical level. A solution may be at hand by constructing effective Lagrangians with soliton solutions that saturate the Bogomol'nyi bound, i.e. so-called Bogomol'nyi-PrasadSommerfield type (BPS) Skyrmions, since their classical static energy grows linearly with the baryon number $A$ (or atomic number) much like the nuclear mass. Support for this idea comes from a recent result from Sutcliffe [10] who found that BPS-type Skyrmions seem to emerge for the original Skyrme model when a large number of vector mesons are added. The additional degrees of freedom cause the mass of the soliton to decrease down to the saturation of the Bogomol'nyi bound. A different and more direct approach was proposed by Adam, Sanchez-Guillen, and Wereszczynski (ASW) 11] by means of a prototype model consisting of only two terms: one of order six in derivatives of the pion fields [12] and a second term, called the potential, which is chosen to be the customary mass term for pions in the Skyrme model [13]. The model leads to BPS-type compacton solutions with size and mass growing as $A^{\frac{1}{3}}$ and $A$ respectively, a result in general agreement with experimental observations. However, the connection between the ASW model and pion physics, or the Skyrme model, is more obscure due to the absence of the nonlinear $\sigma$ and so-called Skyrme terms which are of order 2 and 4 in derivatives, respectively.

Following this picture, some of us 21] have reexamined a more realistic generalization of the Skyrme model which includes terms up to order six in derivatives [12] in the sector where the nonlinear $\sigma$ and Skyrme terms are small. In that limit and for an appropriate choice of mass term, it is possible to find well-behaved analytical solutions for the static solitons. Since they saturate the Bogomol'nyi bound, their static energy is directly proportional to $A$ and we recover some of the results in Ref. [11]. In fact, these solutions allow computing analytically the mass of the nuclei (static and rotational energy) in the regime where quadratic and quartic terms are small perturbations. Adjusting 
the four parameters of the model to fit the resulting binding energies per nucleon with respect to the experimental data of the most abundant isotopes leads to an impressive agreement.

These results support the idea of a BPS-type Skyrme model as the dominant contribution to an effective theory for the properties of nuclear matter. However, a few issues remain to be addressed before such a model is considered viable. We shall concentrate on two of them in this work. First, as for most extensions of the Skyrme model, the BPS-type models in Refs. [11] and 21] generate shell-like configurations for the energy and baryon densities as opposed to what experimental data suggests, i.e. almost constant densities in the nuclei. We show here that it is possible to construct an effective Lagrangian which leads to nonshell configurations and still preserves the agreement with nuclear data. The second issue concerns the inclusion of the Coulomb energy and the isospin symmetry breaking term in the calculation of nuclear masses. In the context of the Skyrme model, these contributions have been thoroughly studied for $A=1$ [14 20] but are usually neglected, to a first approximation, for higher $A$ since they are not expected to overcome the binding energies which are usually large and also because finding the configurations is already numerically challenging so that only small $A$ solutions are known (e.g. approximate toroidal, tetrahedral, cubic configurations for $A=1,2,3$ standard Skyrmions, respectively). However, for our type of near-BPS model, they may have a significant impact on the predictions given the already good agreement with data. Moreover, it turns out that the calculation of the Coulomb energy is simplified by the axial symmetry of the solutions and is calculable for all $A$.

\section{THE NEAR-BPS SKYRME MODEL}

We propose to study the model based on the Lagrangian density

$$
\mathcal{L}=\mathcal{L}_{0}+\mathcal{L}_{2}+\mathcal{L}_{4}+\mathcal{L}_{6}
$$

with

$$
\begin{aligned}
& \mathcal{L}_{0}=-\mu^{2} V(U) \\
& \mathcal{L}_{2}=-\alpha \operatorname{Tr}\left[L_{\mu} L^{\mu}\right] \\
& \mathcal{L}_{4}=\beta \operatorname{Tr}\left(\left[L_{\mu}, L_{\nu}\right]^{2}\right) \\
& \mathcal{L}_{6}=-\frac{3}{2} \frac{\lambda^{2}}{16^{2}} \operatorname{Tr}\left(\left[L_{\mu}, L_{\nu}\right]\left[L^{\nu}, L^{\lambda}\right]\left[L_{\lambda}, L_{\mu}\right]\right)
\end{aligned}
$$

where $L_{\mu}=U^{\dagger} \partial_{\mu} U$ is the left-handed current of the meson fields represented by the $S U(2)$ matrix $U=\phi_{0}+i \tau_{i} \phi_{i}$ which obey the nonlinear condition $\phi_{0}^{2}+\phi_{i}^{2}=1$.

The constants $\mu, \alpha, \beta$, and $\lambda$ are left as free parameters of the model although we shall be interested in the regime where $\alpha$ and $\beta$ are small. The original Skyrme model was built out of only the nonlinear $\sigma$ term, $\mathcal{L}_{2}$, and the Skyrme term, $\mathcal{L}_{4}$. One often adds the so-called mass term, $\mathcal{L}_{0}$, to take into account chiral symmetry breaking and generate a pion mass term for small fluctuations of the chiral field in $V(U)$. Finally, the term of order six in derivatives of the pion fields $\mathcal{L}_{6}$ is equivalent to

$$
\mathcal{L}_{J 6}=-\frac{\varepsilon_{6}}{4} \mathcal{B}^{\mu} \mathcal{B}_{\mu}
$$

that was first proposed by Jackson et al. [12] to allow for the possibility of $\omega$-meson interactions. Here, we define the topological (baryon) current density $\mathcal{B}^{\mu}$ :

$$
\mathcal{B}^{\mu}=\frac{\epsilon^{\mu \nu \rho \sigma}}{24 \pi^{2}} \operatorname{Tr}\left(L_{\nu} L_{\rho} L_{\sigma}\right) .
$$

The boundary condition at infinity must be constant to ensure that solutions for the Skyrme field have finite energy but it also characterizes solutions by a conserved topological charge,

$$
A=\int d^{3} r \mathcal{B}^{0}=-\frac{\epsilon^{i j k}}{24 \pi^{2}} \int d^{3} r \operatorname{Tr}\left(L_{i} L_{j} L_{k}\right) .
$$

The static energy arising from $\mathcal{L}_{6}$ comes from the square of the baryon density

$$
E_{6}=\frac{\varepsilon_{6}}{4} \int\left(\mathcal{B}^{0}(\mathbf{r})\right)^{2} d^{3} r
$$


so in a sense, it is the analog of the Coulomb energy

$$
E_{C}=\frac{1}{2} \int \frac{\rho(\mathbf{r}) \rho\left(\mathbf{r}^{\prime}\right)}{4 \pi\left|\mathbf{r}-\mathbf{r}^{\prime}\right|} d^{3} r d^{3} r^{\prime}
$$

except that instead of following the $\left|\mathbf{r}-\mathbf{r}^{\prime}\right|^{-1}$ law, the interaction is replaced by a $\delta$-function

$$
E_{6}=\frac{\varepsilon_{6}}{4} \int \mathcal{B}^{0}(\mathbf{r}) \mathcal{B}^{0}\left(\mathbf{r}^{\prime}\right) \delta^{3}\left(\mathbf{r}-\mathbf{r}^{\prime}\right) d^{3} r d^{3} r^{\prime}
$$

In other words, the baryonic charge interacts locally.

Historically, $\mathcal{L}_{0}$ and $\mathcal{L}_{6}$ were introduced to provide a more general effective Lagrangian than the original Skyrme model and indeed, the Lagrangian in (1) represents the most general $S U(2)$ model with at most two time derivatives. As an effective theory based on the $1 / N_{c}$ expansion of QCD, there no reason to believe that higher-order derivatives should be absent. However, since one generally relies on the standard Hamiltonian interpretation for the quantization procedure, higher-order time derivatives are usually avoided.

As a result, the model has been studied extensively but remarkably, this was done only for values of parameters $\mu$, $\alpha, \beta$, and $\lambda$ close to that of the original Skyrme model. Presumably these choices were made so that $\mathcal{L}_{2}$ and $\mathcal{L}_{4}$ would continue to have a significant contribution to the mass of the baryons and thereby preserve the relative successes of the Skyrme model in predicting nucleon properties and their link to pion physics ( $\alpha$ is proportional to the pion decay constant $F_{\pi}$ ). Yet this sector of the theory fails to provide an accurate description of the binding energy of heavy nuclei.

Noting that this caveat may come from the fact that the solitons of the Skyrme model do not saturate the Bogomol'nyi bound, ASW proposed a model [11] (equivalent to setting $\alpha=\beta=0$ ) whose solutions are BPS-type solitons and have lower binding energies. A more realistic approach was proposed in Ref. [21] to analyze the full Lagrangian (1) in the sector where $\alpha$ and $\beta$ are relatively small treating these two terms as perturbations. However, in spite of a very good agreement with experimental nuclear masses, there remains an obstacle to the acceptance of such model. Nuclear matter is believed to be uniformly distributed inside a nucleus whereas the solutions of the aforementioned models display shell-like configuration for the baryon and energy densities. Part of this work is to demonstrate that it is possible to construct an effective Lagrangian which leads to nonshell configuration and still preserves and even improves the agreement with nuclear mass data.

We may write the general static solution as

$$
U=e^{i \mathbf{n} \cdot \tau F}=\cos F+i \mathbf{n} \cdot \tau \sin F
$$

where $\hat{\mathbf{n}}$ is the unit vector

$$
\hat{\mathbf{n}}=(\sin \Theta \cos \Phi, \sin \Theta \sin \Phi, \cos \Theta)
$$

Let us consider the model in (11) in the limit where $\alpha$ and $\beta$ are small. For that purpose, we introduce the axial solutions for the $\alpha=\beta=0$ case,

$$
F=F(r), \quad \Theta=\theta, \quad \Phi=n \phi
$$

where $n$ is an integer. The static energy arising from 1 becomes

$$
\begin{aligned}
E_{\text {stat }} & =4 \pi \int r^{2} d r\left(\mu^{2} V+\frac{9 \lambda^{2}}{16} n^{2} F^{\prime 2} \frac{\sin ^{4} F}{r^{4}}\right. \\
& +2 \alpha\left[F^{\prime 2}+\left(n^{2}+1\right) \frac{\sin ^{2} F}{r^{2}}\right] \\
& \left.+16 \beta \frac{\sin ^{2} F}{r^{2}}\left[\left(n^{2}+1\right) F^{\prime 2}+n^{2} \frac{\sin ^{2} F}{r^{2}}\right]\right)
\end{aligned}
$$

Here $F^{\prime}=\partial F / \partial r$ and the topological charge simplifies to

$$
A=-\frac{2 n}{\pi} \int F^{\prime} \sin ^{2} F d r=n
$$

Minimizing of the static energy for $\alpha=\beta=0$ leads to the differential equation for $F$ :

$$
\frac{9 \lambda^{2} n^{2}}{4} \frac{\sin ^{2} F}{2 r^{2}} \partial_{r}\left(\frac{\sin ^{2} F}{r^{2}} F^{\prime}\right)-\mu^{2} \frac{\partial V}{\partial F}=0
$$


The change of variable $z=\frac{2 \sqrt{2} \mu r^{3}}{9 n \lambda}$ allows this last expression to be written in a simple form

$$
\sin ^{2} F \partial_{z}\left[\sin ^{2} F\left(\partial_{z} F\right)\right]-\frac{\partial V}{\partial F}=0
$$

that can be integrated

$$
\frac{1}{2} \sin ^{4} F\left(F_{z}\right)^{2}=V
$$

Regrouping the terms, we get

$$
\int d F \frac{\sin ^{2} F}{\sqrt{2 V}}= \pm\left(z-z_{0}\right)
$$

where $z_{0}$ is an integration constant. Finally, the expression for $F(z)$ can be found analytically provided the integral on the left-hand side is an invertible function of $F$.

The potential (or so-called mass term) $V$ in (17) is a key ingredient in the determination of the solution here. Unfortunately, its exact form is unknown and indeed, has been the object of several discussions [5, 7, 13]. For simplicity, it is often assumed to be

$$
V=\frac{1}{4} \operatorname{Tr}\left[U+U^{\dagger}-2\right]=1-\cos F
$$

This form was considered in ASW for $\alpha=\beta=0$ in the context of BPS-Skyrmions and solving (17) for $F$ led to a BPS-compacton $F(r)=2 \arccos \left(\nu^{1 / 3} r\right)$ for $r \in\left[0, \nu^{-\frac{1}{3}}\right]$, where $\nu=\frac{\mu}{18 n \lambda}$ is a constant depending on the parameters $\lambda, \mu$, and $n$. Note that $F^{\prime}$ diverges as $r \rightarrow \nu^{-\frac{1}{3}}$ and vanishes at $r=0$. Since this solution saturates the Bogomol'nyi bound, the static energy is proportional to the baryon number $A=n$.

A more general choice was introduced in Ref. [5]:

$$
-\mu^{2} V=\sum_{k=1}^{4} C_{k} \operatorname{Tr}\left[U^{k}+U^{\dagger k}-2\right]
$$

This form allows one to recover the chiral symmetry breaking pion mass term $-\frac{1}{2} m_{\pi}^{2} \pi \cdot \pi$ in the limit of small pion field fluctuations $U=2 \exp \left(i \tau_{a} \pi_{a} / F_{\pi}\right)$ and to find a relation between the pion mass $m_{\pi}$ and the parameter $\mu$,

$$
\sum_{k=1}^{\infty} k^{2} C_{k}=-\frac{m_{\pi}^{2} F_{\pi}^{2}}{16}
$$

The case considered in Ref. [21] is a particular example of such potential with

$$
-C_{1}=C_{2}=C_{3}=4 C_{4}=\frac{\mu^{2}}{128}
$$

and $C_{k>4}=0$. Assuming the axial solution (11), the potential simplifies to

$$
V=\sin ^{2}\left(\frac{F}{2}\right) \cos ^{6}\left(\frac{F}{2}\right) .
$$

and upon integration (17), we get the solution

$$
F(r)=\mp 2\left|\arccos \left(e^{-\nu r^{3}}\right)\right|
$$

with $\nu=\frac{\mu}{18 n \lambda}$. Here, we use the absolute value in order to eliminate of the sign ambiguity of the arccos function. In order to set the baryon number to $|A|=n$ and the integration constant $z_{0}$, we fix the boundary conditions $F(0)=0$ and $F(\infty)=\mp \pi$ for positive and negative baryon number respectively. Note that the exponential fall off of $F$ at large $r$ helps prevent some quantities such as the moments of inertia from becoming infinite.

Unfortunately, the BPS-type models in Refs. [11] and 21] both lead to shell configurations for the baryon and energy densities which disagrees with experimental results. This is often the case for Skyrme models and it is clear 
from expressions (12) and (13) that this behavior can be traced back to the form of the profile $F(r)$ or more precisely to the derivative $F^{\prime}(r)$ which tends to zero near $r=0$ for such models.

Let us consider the more appropriate solution of the form

$$
F(r)=\mp 2\left|\arccos \left(e^{-a r^{2}}\right)\right|
$$

with $a=\nu^{\frac{2}{3}}$ and similar boundary conditions $F(0)=0$ and $F(\infty)=\mp \pi$ Here, since $F^{\prime}(0) \neq 0$, neither the baryon density

$$
\mathcal{B}^{0}(r)=-\frac{n}{2 \pi^{2}} \frac{\sin ^{2} F}{r^{2}} F^{\prime}=\frac{2 a n}{\pi^{2}} \frac{e^{-a r^{2}}}{r} \sqrt{1-e^{-2 a r^{2}}}
$$

nor the static energy density vanishes near $r=0$. We find by inspection of (17), that this solution emerges from a potential similar to (21), namely

$$
V=-\frac{8 \sin ^{2}\left(\frac{F}{2}\right) \cos ^{6}\left(\frac{F}{2}\right)}{9 \ln \left(\cos ^{2}\left(\frac{F}{2}\right)\right)} .
$$

The logarithmic dependence in the denominator of this expression could be problematic since $F=0$ at $r=0$ and $F=\mp \pi$ at $r=\infty$ but the limits for $V$ are well defined and finite, i.e. $\lim _{r \rightarrow 0, \infty} V=\frac{8}{9}, 0$ respectively. It is interesting to note that according to (16), the square root of the potential

$$
\sqrt{V}=\frac{3 \lambda \pi}{8 \mu}\left(-\frac{2 n}{\pi} \frac{\sin ^{2} F}{r^{2}} F^{\prime}\right)=\frac{3 \lambda \pi}{8 \mu} \mathcal{B}^{0}(r)
$$

corresponds to the baryon radial density (the term in parenthesis) up to a multiplicative constant. Thus, in order to obtain a nonshell baryon density, it suffices to construct a potential $V$ that does not vanish at small $r$. Such a potential would also imply that $F^{\prime}(0) \neq 0$. Our choice of potential clearly verifies this requirement but this relation also explains why the earlier BPS-type models could not generate a nonshell configuration, namely $V \sim\left(1-\cos \left(\frac{F}{2}\right)\right)$ and $F^{\prime}(0)=0$ in that limit.

The expression (25) only applies to the axial solution (11) and we need to write a more general form for $V$ in terms of $U$ if this is to be used in the expression for the Lagrangian. A simple but not unique approach to construct the potential is to identify $\cos (F / 2)$ to the expression

$$
\frac{1}{4}\left(2 I+U+U^{\dagger}\right)=\cos ^{2}\left(\frac{F}{2}\right) I
$$

where $I$ is the identity matrix. Then, a convenient expression for $V$ is given by

$$
-\mu^{2} V(U)=\frac{\mu^{2}}{576} \operatorname{Tr}\left[\frac{\left(2 I-U-U^{\dagger}\right)\left(2 I+U+U^{\dagger}\right)^{3}}{\ln \left(\left(2 I+U+U^{\dagger}\right) / 4\right)}\right]
$$

Comparing this expression to (18) allows retrieving each coefficient

$$
\begin{aligned}
& C_{1}=-0.129631 \mu^{2}, \quad C_{2}=-0.100632 \mu^{2}, \quad C_{3}=-0.045532 \mu^{2}, \\
& C_{4}=-0.0105061 \mu^{2}, \ldots
\end{aligned}
$$

such that

$$
\sum_{k=1}^{\infty} k^{2} C_{k}=-1.12798 \mu^{2}=-\frac{m_{\pi}^{2} F_{\pi}^{2}}{16}
$$

Inserting expression (23) in (12), we get the static energy of the soliton in the small $\alpha$ and $\beta$ approximation

$$
E_{\text {stat }}=E_{0}+E_{2}+E_{4}+E_{6}
$$


with

$$
\begin{aligned}
& E_{0}=\frac{4}{3} \sqrt{2}(-3+2 \sqrt{3}) n \pi^{3 / 2} \lambda \mu \\
& E_{2}=\left(8(\sqrt{2}-1)\left(n^{2}+1\right)+6 \sqrt{2} \zeta\left(\frac{5}{2}\right)\right) \pi^{3 / 2} \alpha \nu^{-1 / 3} \\
& E_{4}=64\left(2(16 \sqrt{2}(\sqrt{3}-1)-15) n^{2}+2\right) \pi^{3 / 2} \beta \nu^{1 / 3} \\
& E_{6}=\frac{4}{3} \sqrt{2}(-3+2 \sqrt{3}) n \pi^{3 / 2} \lambda \mu
\end{aligned}
$$

where $\nu=\frac{\mu}{18 n \lambda}$ sets the scale of the solution and $\zeta$ is the Riemann $\zeta$ - function . The terms $V$ and $E_{6}$ are proportional to the baryon number $A=n$ as one expects from solutions that saturate the Bogomol'nyi bound whereas the small perturbations $E_{2}=A^{1 / 3}\left(a_{2}+b_{2} A^{2}\right)$ and $E_{4}=A^{-1 / 3}\left(a_{4}+b_{4} A^{2}\right)$ have a more complex dependence. Part of this behavior, the overall factor $A^{ \pm 1 / 3}$, is due to the scaling. The additional factor of $A^{2}$ comes from the axial symmetry of the solution (11). Note that it is also easy to calculate analytically the root mean square radius of the baryon density

$$
\left\langle r^{2}\right\rangle^{\frac{1}{2}}=\frac{1}{2}\left(\frac{18 A \lambda}{\mu}\right)^{\frac{1}{3}} \sqrt{-1+\ln 16}
$$

which is consistent with experimental observation for the charge distribution of nuclei $\left\langle r^{2}\right\rangle^{\frac{1}{2}}=r_{0} A^{\frac{1}{3}}$.

In order to represent physical nuclei, we have taken into account their rotational and isorotational degrees of freedom and quantize the solitons. The standard procedure is to use the semiclassical quantization which is described in the next section.

\section{QUANTIZATION}

Skyrmions are not pointlike particles. So we resort to a semiclassical quantization method which consists in adding an explicit time dependence to the zero modes of the Skyrmions and applying a time-dependent (iso)rotations on the Skyrme fields by $S U(2)$ matrix $A(t)$ and $B(t)$

$$
\tilde{U}(\mathbf{r}, t)=A(t) U(R(B(t)) \mathbf{r}) A(t)
$$

where $R(B(t))$ is the associated $S O(3)$ rotation matrix. The approach assumes that the Skyrmion behave as a rigid rotator. Upon insertion of this ansatz in the time-dependent part of the full Lagrangian (1), we can write the (iso)rotational Lagrangian as

$$
\mathcal{L}_{\text {rot }}=\frac{1}{2} a_{i} U_{i j} a_{j}-a_{i} W_{i j} b_{j}+\frac{1}{2} b_{i} V_{i j} b_{j},
$$

where $a_{i}=-i \operatorname{Tr} A^{\dagger} \dot{A}$ and $b_{i}=i \operatorname{Tr} \dot{B} B^{\dagger}$

The moment of inertia tensors $U_{i j}$ is given by

$$
\begin{aligned}
U_{i j} & =\int d^{3} r \mathcal{U}_{i j}=-\int d^{3} r\left[2 \alpha \operatorname{Tr}\left(T_{i} T_{j}\right)\right. \\
& +4 \beta \operatorname{Tr}\left(\left[L_{p}, T_{i}\right]\left[L_{p}, T_{j}\right]\right) \\
& \left.+\frac{9 \lambda^{2}}{16^{2}} \operatorname{Tr}\left(\left[T_{i}, L_{p}\right]\left[L_{p}, L_{q}\right]\left[L_{q}, T_{j}\right]\right)\right]
\end{aligned}
$$

where $T_{i}=i U^{\dagger}\left[\frac{\tau_{i}}{2}, U\right]$. The expressions for $W_{i j}$ and $V_{i j}$ are similar except that the isorotational operator $T_{i}$ is replaced by a rotational analog $S_{i}=-\epsilon_{i k l} x_{k} L_{l}$ as follows:

$$
\begin{aligned}
W_{i j} & =\int d^{3} r \mathcal{W}_{i j}=\int d^{3} r \mathcal{U}_{i j}\left(T_{j} \rightarrow S_{j}\right) \\
V_{i j} & =\int d^{3} r \mathcal{V}_{i j}=\int d^{3} r \mathcal{U}_{i j}\left(T_{j} \rightarrow S_{j}, T_{i} \rightarrow S_{i}\right) .
\end{aligned}
$$


Following the calculations in 21] for axial solution of the form (11), we find that all off-diagonal elements of the inertia tensors vanish. Furthermore, one can show that $U_{11}=U_{22}$ and $U_{33}$ can be obtained by setting $n=1$ in the expression for $U_{11}$. Similar identities hold for $V_{i j}$ and $W_{i j}$ tensors. The axial symmetry of the solution imposes the constraint $L_{3}+n K_{3}=0$ which is simply the statement that a spatial rotation by an angle $\theta$ about the axis of symmetry can be compensated by an isorotation of $-n \theta$ about the $\tau_{3}$ axis. It follows from expressions $(31)-(33)$ that $W_{11}=W_{22}=0$ for $|n| \geq 2$ and $n^{2} U_{33}=n W_{33}=V_{33}$.

The general form of the rotational Hamiltonian is given by [22]

$$
H_{\mathrm{rot}}=\frac{1}{2}\left[\frac{\left(L_{1}+W_{11} \frac{K_{1}}{U_{11}}\right)^{2}}{V_{11}-\frac{W_{11}^{2}}{U_{11}}}+\frac{\left(L_{2}+W_{22} \frac{K_{2}}{U_{22}}\right)^{2}}{V_{22}-\frac{W_{22}^{2}}{U_{22}}}+\frac{\left(L_{3}+W_{33} \frac{K_{3}}{U_{33}}\right)^{2}}{V_{33}-\frac{W_{33}^{2}}{U_{33}}}+\frac{K_{1}^{2}}{U_{11}}+\frac{K_{2}^{2}}{U_{22}}+\frac{K_{3}^{2}}{U_{33}}\right]
$$

where $\left(K_{i}\right) L_{i}$ the body-fixed (iso)rotation momentum canonically conjugate to $\left(a_{i}\right) b_{i}$. The expression for the rotational energy of the nucleon $A=1$ simplifies due to the spherical symmetry

$$
E_{\mathrm{rot}}^{N}=\frac{3}{8 U_{11}}
$$

It is also easy to calculate the rotational energies for nuclei with winding number $|n| \geq 2$

$$
H_{\mathrm{rot}}=\frac{1}{2}\left[\frac{\mathbf{L}^{2}}{V_{11}}+\frac{\mathbf{K}^{2}}{U_{11}}+\xi K_{3}^{2}\right]
$$

with

$$
\xi=\frac{1}{U_{33}}-\frac{1}{U_{11}}-\frac{n^{2}}{V_{11}}
$$

These momenta are related to the usual space-fixed isospin (I) and spin (J) by the orthogonal transformations

$$
\begin{gathered}
I_{i}=-R\left(A_{1}\right)_{i j} K_{j}, \\
J_{i}=-R\left(A_{2}\right)_{i j}^{\mathrm{T}} L_{j} .
\end{gathered}
$$

According to (37) and (38), we see that the Casimir invariants satisfy $\mathbf{K}^{2}=\mathbf{I}^{2}$ and $\mathbf{L}^{2}=\mathbf{J}^{2}$ so the rotational Hamiltonian is given by

$$
H_{\mathrm{rot}}=\frac{1}{2}\left[\frac{\mathbf{J}^{2}}{V_{11}}+\frac{\mathbf{I}^{2}}{U_{11}}+\xi K_{3}^{2}\right] .
$$

We are looking for the lowest eigenvalue of $H_{\text {rot }}$ which depends on the dimension of the spin and isospin representation of the eigenstate $\left|i, i_{3}, k_{3}\right\rangle\left|j, j_{3}, l_{3}\right\rangle$. For $\alpha=\beta=0$, we can show that $\xi$ is negative and we shall assume that this remains true for small values of $\alpha$ and $\beta$. Then, for a given spin $j$ and isospin $i, \kappa$ must take the largest possible eigenvalue $k_{3}$. Since $\mathbf{K}^{2}=\mathbf{I}^{2}$ and $\mathbf{L}^{2}=\mathbf{J}^{2}$, the state with highest weight is characterized by $k_{3}=i$ and $l_{3}=j$ and since nuclei are build out of $A$ fermions we must have an isospin $j \leq A / 2$. On the other hand, the axial symmetry of the static solutions implies that $k_{3}=-l_{3} / n$ where $n=A$. But for even $A$ nuclei, $k_{3}$ must be an integer and $\left|l_{3} / n\right| \leq|j / n| \leq|A /(2 n)|=1 / 2$ so

$$
0 \leq\left|k_{3}\right| \leq\left[\left|\frac{A}{2 n}\right|\right]=0
$$

Similarly for half-integer spin nuclei, $\left|k_{3}\right|$ must be a half-integer so the only possible value is

$$
\frac{1}{2} \leq\left|k_{3}\right| \leq\left|\frac{A}{2 n}\right|=\frac{1}{2}
$$

Summarizing, if we assume for simplicity that the $\alpha$ and $\beta$ terms only generate small perturbations, the largest possible eigenvalue $k_{3}$ is

$$
\kappa=\max \left(\left|k_{3}\right|\right)=\left\{\begin{array}{ll}
0 & \text { for } A=\text { even } \\
\frac{1}{2} & \text { for } A=\text { odd }
\end{array} .\right.
$$


The lowest eigenvalue of the rotational Hamiltonian $H_{\text {rot }}$ for a nucleus is then given by [21]

$$
E_{\mathrm{rot}}=\frac{1}{2}\left[\frac{j(j+1)}{V_{11}}+\frac{i(i+1)}{U_{11}}+\xi \kappa^{2}\right]
$$

The spin of the most abundant isotopes is fairly well known. The isospins are not so well known so we resort to the usual assumption that the most abundant isotopes correspond to states with lowest isorotational energy. Since $i \geq\left|i_{3}\right|$, the lowest value that $i$ can take is simply $\left|i_{3}\right|$ where $i_{3}=A / 2-Z$. For example, the deuteron corresponds to $A=n=2, i=0, j=1$, and $\kappa=0$, so the rotational energy reduces to

$$
E_{\text {rot }}^{D}=\frac{1}{V_{11}}
$$

The explicit calculations of the rotational energy of nuclei then require only three moments of inertia which can be found analytically:

$$
\begin{aligned}
U_{11}= & \frac{\pi^{3 / 2}}{18}\left[24(2 \sqrt{2}-1) \alpha \nu^{-1}\right. \\
+ & 128\left(2 \sqrt{2}(3-4 \sqrt{3})\left(3 n^{2}+1\right)+3\left(12 n^{2}+7\right)\right) \beta \nu^{-1 / 3} \\
+ & \left.3 \sqrt{2}(8 \sqrt{3}-9)\left(3 n^{2}+1\right) \lambda^{2} \nu^{1 / 3}\right] \\
V_{11} & =\frac{\pi^{3 / 2}}{18}\left[6(2 \sqrt{2}-1)\left(n^{2}+3\right) \alpha \nu^{-1}\right. \\
& +32\left(32 \sqrt{2}(3-4 \sqrt{3}) n^{2}+3\left(67 n^{2}+9\right)\right) \beta \nu^{-1 / 3} \\
& \left.+12 \sqrt{2}(8 \sqrt{3}-9) n^{2} \lambda^{2} \nu^{1 / 3}\right]
\end{aligned}
$$

and $U_{33}=U_{11}(n \rightarrow 1)$.

So far, both contributions to the mass of the nucleus, $E_{\text {stat }}$ and $E_{\text {rot }}$, are charge invariant. Since this is a symmetry of the strong interaction, it is reflected in the construction of the Lagrangian (11) and one expects that the two terms form the dominant portion of the mass. However, isotope masses differ by a few percent so this symmetry is broken for physical nuclei. In the next section, we consider two additional contributions to the mass, the Coulomb energy associated with the charge distribution inside the Skyrmion and an isospin breaking term that may be attributed to the up and down quark mass difference.

\section{COULOMB ENERGY AND ISOSPIN BREAKING}

Even if we thought of a nucleus as a simple collection of individual protons and neutrons, there would be a repulsive electromagnetic force between protons and the process would require energy to bring these charges together. The result is an increase in the mass of the object by an amount corresponding to the Coulomb energy. Such an effect is of course also present in the Skyrmions description of nuclei since the static configuration has non-vanishing charge density. The electromagnetic and isospin breaking contributions to the mass have been thoroughly studied for $A=1$, mostly in the context of the computation of the proton-neutron mass difference [14 20], but are usually neglected, to a first approximation, for higher $A$ since they are not expected to overcome the large binding energies predicted by the model. There are also practical reasons why they are seldom taken into account. The higher baryon number configurations of the original Skyrme model are nontrivial (toroidal shape for $A=2$, tetrahedral for $A=3$, etc.) and finding them exactly either requires heavy numerical calculations (see for example [23]) or some kind of clever approximation like rational maps [24]. Moreover, the computation of the Coulomb energy is more challenging in general since it involves two integrations over volume. One can also argue that the Coulomb energy of Skyrmions is somewhat reduced by shell-like configurations of the charge densities as opposed to what it would be for a nearly constant spherical density found in electron scattering experiments. In our case however, we are interested in a more precise calculation of the nuclei masses and an estimate of the Coulomb energy is desirable, and even more so in our model which generates nonshell configurations. It turns out that the analytical form of the chiral angle $F(r)$ in (23) and the axial symmetry of the solution simplify the computation of the Coulomb energy. 
Let us first consider the charge density inside Skyrmions. Following Adkins et al. [4], we write the electromagnetic current

$$
J_{E M}^{\mu}=\frac{1}{2} \mathcal{B}^{\mu}+J_{V}^{\mu 3}
$$

with $\mathcal{B}^{\mu}$ the baryon density and $J_{V}^{\mu 3}$ the vector current density, so the conserved electric charge is given by

$$
Z=\int d^{3} r J_{E M}^{0}=\int d^{3} r\left(\frac{1}{2} \mathcal{B}^{0}+J_{V}^{03}\right)=\frac{A}{2}+k_{3}
$$

with $k_{3}$, the eigenvalue of third component of isospin in the body-fixed frame. The vector current is then defined as the sum of the left and right handed currents

$$
J_{V}^{\mu i}=J_{R}^{\mu i}+J_{L}^{\mu i}
$$

which are invariant under $S U(2)_{L} \otimes S U(2)_{R}$ transformations of the form $U \rightarrow L U R^{\dagger}$. More explicitly, we get

$$
J_{V}^{0 i}=\mathcal{U}_{i j} a_{j}-\mathcal{W}_{i j} b_{j}
$$

where $\mathcal{U}_{i j}$ and $\mathcal{W}_{i j}$ are the moment of inertia densities in (31)-(33). In the quantized version, $a_{j}$ and $b_{j}$ are expressed in terms of the conjugate operators $K_{i}$ and $L_{i}$. Here we only need the relation

$$
K_{i}=U_{i j} a_{j}-W_{i j} b_{j}
$$

Since the off-diagonal elements of $U_{i j}$ and $W_{i j}$ vanish when the solution is axially symmetric and also $n^{2} U_{33}=n W_{33}=$ $V_{33}$, we have

$$
a_{3}=\frac{K_{3}+W_{33} b_{3}}{U_{33}}=\frac{K_{3}}{U_{33}}+n b_{3}
$$

Inserting $a_{3}$ in (47), the isovector electric current density reduces to

$$
J_{V}^{03}=K_{3} \frac{\mathcal{U}_{33}}{U_{33}}
$$

where $\mathcal{U}_{33} / U_{33}$ may be interpreted here as a normalized moment of inertia density for the third component of isospin. Finally, the electric charge density is given by

$$
\rho(\mathbf{r}) \equiv \frac{1}{2} \mathcal{B}^{0}(\mathbf{r})+i_{3} \frac{\mathcal{U}_{33}(\mathbf{r})}{U_{33}}
$$

where we have replaced $k_{3}$ by $i_{3}$ using the fact that the charge density of body-fixed and space-fixed frame only differs by a rotation.

The Coulomb energy stored in a charge distribution $\rho(\mathbf{r})$ takes the usual form (8). In practice, unless one considers very simple configurations, it is not possible to find an analytical expression for the Coulomb energy. Nonetheless, it is often helpful to expand $\rho(\mathbf{r})$ in terms of normalized spherical harmonics to take care of the angular integrations

$$
\rho(\mathbf{r})=\sum_{l, m} \rho_{l m}(r) Y_{l}^{m *}(\theta, \phi)
$$

Following the approach described in [25], we define the quantities

$$
Q_{l m}(r)=\int_{0}^{r} d r^{\prime} r^{l+2} \rho_{l m}\left(r^{\prime}\right)
$$

which, at large distance, are equivalent to a multipole moments of the distribution. Then, each moment contributes to the Coulomb energy by an amount

$$
U_{l m}=\frac{1}{2 \epsilon_{0}} \int_{0}^{\infty} d r r^{-2 l-2}\left|Q_{l m}(r)\right|^{2}
$$


and the total Coulomb energy associated to the distribution is given by

$$
E_{C}=\sum_{l=0}^{\infty} \sum_{m=-l}^{l} U_{l m}
$$

In our case, the angular dependence of the charge density is rather simple. The first part is a spherically symmetric contribution

$$
\mathcal{B}^{0}(r)=-\frac{n}{2 \pi^{2}} \frac{\sin ^{2} F}{r^{2}} F^{\prime}
$$

whereas the only non-trivial piece comes from the third moment density $\mathcal{U}_{33}(\mathbf{r})$ and is proportional to $\sin ^{2}(\theta)$

$$
\mathcal{U}_{33}=\left(4 \alpha \sin ^{2} F+32 \beta \sin ^{2} F\left(F^{\prime 2}+\frac{\sin ^{2} F}{r^{2}}\right)+\frac{9 \lambda^{2}}{8} F^{\prime 2} \sin ^{2} F\left(\frac{\sin ^{2} F}{r^{2}}\right)\right) \sin ^{2} \theta=u_{33}(r) \sin ^{2} \theta
$$

The summation (49) consists of only two terms

$$
\begin{aligned}
& \rho_{00}(r)=2 \sqrt{\pi} \frac{\mathcal{B}^{0}(r)}{2}+\frac{4 \sqrt{\pi}}{3} \frac{u_{33}(r)}{U_{33}} i_{3} \\
& \rho_{20}(r)=-\frac{4}{3} \sqrt{\frac{\pi}{5}} \frac{u_{33}(r)}{U_{33}} i_{3}
\end{aligned}
$$

The expressions for moments $Q_{00}(r)$ and $Q_{20}(r)$ are found by integrating (50) analytically. Finally, we obtain the Coulomb energy by computing numerically the last remaining integral

$$
E_{C}=\frac{1}{2 \epsilon_{0}} \int_{0}^{\infty}\left(\left|Q_{00}\right|^{2} r^{-4}+\left|Q_{20}\right|^{2} r^{-8}\right) r^{2} d r
$$

The Coulomb energy alone cannot explain the isotope mass difference. This is particularly evident for $A=1$ where the proton mass is known to be smaller than that of the neutron although the Coulomb energy alone would suggest otherwise. On the other hand, isospin is not an exact symmetry, a fact that may be traced back to the up and down quark mass difference. Several attempts have been made to modelize the isospin symmetry breaking term within the Skyrme model [19, 20]. Here we shall assume for simplicity that this results in a contribution proportional to the third component of isospin

$$
E_{I}=a_{I} i_{3}
$$

with the parameter $a_{I}$ fixed by setting the neutron-proton mass difference to its experimental value. Since both of them have the same static and rotational energies,

$$
\Delta M_{n-p}^{\text {expt }}=\left(E_{C}^{n}-E_{C}^{p}\right)-a_{I}=1.293 \mathrm{MeV}
$$

and

$$
a_{I}=\left(E_{C}^{p}-E_{C}^{n}\right)-\Delta M_{n-p}^{\mathrm{expt}}
$$

Summarizing, the mass of a nucleus reads

$$
E\left(A, i, j, k_{3}, i_{3}\right)=E_{\text {stat }}(A)+E_{\text {rot }}\left(A, i, j, k_{3}\right)+E_{C}\left(A, i_{3}\right)+E_{I}\left(A, i_{3}\right)
$$

where we have written the explicit dependence of each piece in terms of the relevant nuclear quantum numbers of the nuclei. The prediction depends on the parameters of the model $\mu, \alpha, \beta$, and $\lambda$.

\section{RESULTS AND DISCUSSION}

The values of the parameters $\mu, \alpha, \beta$ and $\lambda$ remain to be fixed. Let us first consider the case where $\alpha=\beta=0$. This should provide us with a good estimate for the values of $\mu, \alpha, \beta$, and $\lambda$ required in the 4-parameter model (1) and, 
after all, it corresponds to the limit where the minimization of the static energy leads to the exact analytical solution (23).

We need two input parameters to set $\mu$ and $\lambda$. For simplicity, we choose the mass of the nucleon and that a nucleus $X$ with zero (iso)rotational energy (i.e. a nucleus with zero spin and isospin) and neglect for now the Coulomb and isospin breaking energies. The total energy of these two states is according to (27) and (41)

$$
\begin{aligned}
& E_{N}=\frac{8}{3} \sqrt{2}(-3+2 \sqrt{3}) \pi^{3 / 2} \lambda \mu+\frac{(18)^{4 / 3}}{32 \pi^{3 / 2} \sqrt{2}(8 \sqrt{3}-9)(\lambda \mu)^{1 / 3} \lambda^{4 / 3}} \\
& E_{X}=\frac{8}{3} \sqrt{2}(-3+2 \sqrt{3}) n_{X} \pi^{3 / 2} \lambda \mu
\end{aligned}
$$

Solving for $\lambda$ and $\mu$ we get

$$
\begin{gathered}
\lambda=\frac{3(-3+2 \sqrt{3})^{1 / 4} 3^{3 / 4} n_{X}}{4\left(2 E_{X}\right)^{1 / 4}\left(\pi(8 \sqrt{3}-9)\left(n_{X} E_{N}-E_{X}\right)\right)^{3 / 4}} \\
\mu=\frac{\left(\left(n_{X} E_{N}-E_{X}\right)(8 \sqrt{3}-9)\right)^{3 / 4} E_{X}^{5 / 4}}{(3 \pi)^{3 / 4}(2(2 \sqrt{3}-3))^{5 / 4} n_{X}^{2}}
\end{gathered}
$$

As an example, let us examine the case where the nucleus $X$ is Helium-4, the first doubly magic number nucleus with zero spin and isospin. Setting the mass of the nucleon as the average mass of the proton and neutron i.e. $E_{N}=938.919$ $\mathrm{MeV}$ and that of Helium-4 nucleus to $E_{H e}=3727.38 \mathrm{MeV}$, we get the numerical value $\lambda=0.00641362 \mathrm{MeV}^{-1}$, $\alpha=\beta=0$ and $\mu=14908$. $\mathrm{MeV}^{2}$ which we shall refer as Set I. The masses of the nuclei including static, (iso)rotational, Coulomb, and isospin breaking contributions are then computed using (53). Table I shows the relative deviation of the predicted with regard to experimental values of nuclear masses of a few isotopes (Set I). The predictions are accurate to $0.4 \%$ or better even for heavier nuclei. Part of this accuracy is probably due to the fact that the static energy of a BPS-type solution is proportional to $A$ so if it dominates, the nuclear masses should follow approximately the same pattern. However, the predictions remain surprisingly good for a 2-parameter model. Perhaps more relevant are the predictions of the binding energy per nucleon $(B / A)$. The results are presented in Fig. 1 - Set I (solid line) and can be compared to the experimental values (black circles). We consider here only a subset of the table of nuclei in [26] composed of the most abundant 144 isotopes. We observe a sharp rise of the binding energy per nucleon at small $A$ followed by a slow linear increase for larger nuclei. The overall accuracy is of the order of $15 \%$ which is rather good considering the fact that the calculation involves the mass difference between the nucleus and its constituents.

Experimentally the charge radius of the nucleus is known to behave approximately as

$$
\left\langle r_{\mathrm{em}}^{2}\right\rangle^{\frac{1}{2}}=r_{0} A^{\frac{1}{3}}
$$

with $r_{0}=1.25 \mathrm{fm}$. On the other hand, it is possible to calculate the root mean square radius for the baryon density [see Eq (28)] which leads to

$$
\left\langle r^{2}\right\rangle^{\frac{1}{2}}=(2.599 \mathrm{fm}) A^{\frac{1}{3}}
$$

For the charge radius $\left\langle r_{\mathrm{em}}^{2}\right\rangle^{\frac{1}{2}}$, the dependence on $A$ is more complex since it involves an additional isovector contribution (48)

$$
\left\langle r_{\mathrm{em}}^{2}\right\rangle=\frac{\int d^{3} r r^{2} \rho(\mathbf{r})}{\int d^{3} r \rho(\mathbf{r})}=\frac{A}{2 Z}\left\langle r^{2}\right\rangle+\left\langle r_{V}^{2}\right\rangle
$$

where $Z=i_{3}+A / 2$ is the charge of the nucleus. We get the expression

$$
\begin{aligned}
\left\langle r_{V}^{2}\right\rangle & =\frac{i_{3}}{Z U_{33}} \int d r r^{4} u_{33}(r) \\
& =\frac{i_{3}}{Z U_{33}} \frac{\pi^{3 / 2} \lambda A^{1 / 3}}{24 \mu^{5 / 3}}\left[648 \times 2^{1 / 6} 3^{1 / 3}(\sqrt{2}-8) A^{4 / 3} \alpha \lambda^{2 / 3}\right. \\
& +128(4 \sqrt{2}(-9+16 \sqrt{3})-279) A^{2 / 3} \beta \mu^{2 / 3} \\
& \left.+13 \times 2^{5 / 6} 3^{1 / 6}(9 \sqrt{3}-32) \lambda^{4 / 3} \mu^{4 / 3}\right]
\end{aligned}
$$


where $U_{33}$ also depends on $A$ and is obtained by substituting $U_{33}=U_{11}(n \rightarrow 1)$ in (43). Our computation verifies that the charge radius obeys roughly the proportionality relation

$$
\left\langle r_{\mathrm{em}}^{2}\right\rangle^{\frac{1}{2}} \sim(2.637 \mathrm{fm}) A^{\frac{1}{3}}
$$

but overestimates the experimental value of $r_{0}$ by approximately a factor of 2 .

Let us now release the constraints on $\alpha$ and $\beta$ and allow for small perturbations from the nonlinear $\sigma$ and Skyrme term. In order to estimate the magnitude of the parameters $\alpha$ and $\beta$ in a real physical case, we perform two fits: Set II optimizes the four parameters $\mu, \alpha, \beta$ and $\lambda$ to better reproduce the masses of the nuclei while Set III tries to reach the best agreement with respect to the binding energy per nucleon, $B / A$. Both fits are performed with data from the same subset of the most abundant 144 isotopes as before. A summary of the results is presented in Table I while Fig. 1 displays the general behavior of $B / A$ as a function of the baryon number for Sets I, II, III, and experimental values.

\begin{tabular}{|c|c|c|c|c|}
\hline & Set I & Set II & Set III & Experiment \\
\hline$\mu\left(10^{4} \mathrm{MeV}^{2}\right)$ & 1.49080 & 1.50571 & 1.72955 & \\
\hline$\alpha\left(10^{-3} \mathrm{MeV}^{2}\right)$ & 0 & 5.88118 & 22.0821 & \\
\hline$\beta\left(10^{-6} \mathrm{MeV}^{0}\right)$ & 0 & -1.84877 & -5.80989 & \\
\hline$\lambda\left(10^{-3} \mathrm{MeV}^{-1}\right)$ & 6.41362 & 6.33973 & 5.53691 & \\
\hline$F_{\pi}(\mathrm{MeV})$ & 0 & 0.307 & 0.594 & 186 \\
\hline$m_{\pi}(\mathrm{MeV})$ & - & 208530 & 82300 & 138 \\
\hline$e\left(10^{4}\right)$ & - & -185000 & -5380 & \\
\hline$r_{0}(\mathrm{fm})$ & 2.637 & 2.617 & 2.385 & 1.23 \\
\hline Nucleus X & \multicolumn{3}{|c|}{$\frac{E_{X}-E_{\exp }}{E_{\exp }}$} & $E_{\exp }(\mathrm{MeV})$ \\
\hline Nucleon & Input & -0.0008 & 0.0020 & 938.919 \\
\hline${ }^{2} \mathrm{H}$ & -0.0032 & -0.0048 & -0.0020 & 1875.61 \\
\hline${ }^{3} \mathrm{H}$ & -0.0042 & -0.0057 & -0.0030 & 2808.92 \\
\hline${ }^{4} \mathrm{He}$ & Input & -0.0017 & -0.0009 & 3727.38 \\
\hline${ }^{6} \mathrm{Li}$ & -0.0017 & -0.0034 & -0.0010 & 5601.52 \\
\hline${ }^{7} \mathrm{Li}$ & -0.0014 & -0.0031 & -0.0008 & 6533.83 \\
\hline${ }^{9} \mathrm{Be}$ & -0.0006 & -0.0023 & -0.0001 & 8392.75 \\
\hline${ }^{10} \mathrm{~B}$ & -0.0004 & -0.0021 & -0.00001 & 9324.44 \\
\hline${ }^{16} \mathrm{O}$ & 0.0010 & -0.0008 & 0.0009 & 14895.1 \\
\hline${ }^{20} \mathrm{Ne}$ & 0.0010 & -0.0007 & 0.0008 & 18617.7 \\
\hline${ }^{40} \mathrm{Ca}$ & 0.0016 & 0.0001 & 0.0006 & 37214.7 \\
\hline${ }^{56} \mathrm{Fe}$ & 0.0018 & 0.0001 & 0.0004 & 52089.8 \\
\hline${ }^{238} \mathrm{U}$ & 0.0004 & 0.00001 & 0.0006 & 221696 \\
\hline
\end{tabular}

We find that the two new sets of parameters are very close to Set I. The nonlinear $\sigma$ and Skyrme parameters $\alpha$ and $\beta$ are small in magnitude but in order to make a relevant comparison, it is best to look at the relative importance of the contributions in (11) and how they scale with respect to the parameters of the model, namely

$$
\begin{aligned}
& \lambda \mu: \alpha \nu^{-1 / 3}: \quad \beta \nu^{1 / 3}: \lambda \mu
\end{aligned}
$$

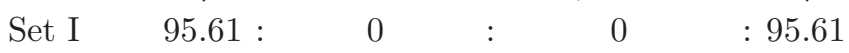

$$
\begin{aligned}
& \text { Set II } \quad 95.46: 4.408 \times 10^{-5}:-2.255 \times 10^{-4}: 95.46 \\
& \text { Set III } 95.74: 1.418 \times 10^{-4}:-7.904 \times 10^{-4}: 95.74
\end{aligned}
$$

for $\mathcal{L}_{0}, \mathcal{L}_{2}, \mathcal{L}_{4}$, and $\mathcal{L}_{6}$ respectively. Clearly, the nonlinear $\sigma$ and Skyrme terms are extremely small compared to that of $\mathcal{L}_{0}$ and $\mathcal{L}_{6}$, i.e. by at least 6 orders of magnitude. This provides support to the assumption that (23) is a good approximation to the exact solution. The overall factor $\lambda \mu$ remains approximately the same for all the sets but $B / A$ turns out to be somewhat sensitive to these small variations because it involves a mass difference. Even more sensitive to small change in parameters is the charge radius $r_{0}$ with $10 \%$ decrease between Set II and Set III (Table I) which suggests that the predicted value of $r_{0}$ should be taken as an estimate rather than a firm prediction. 


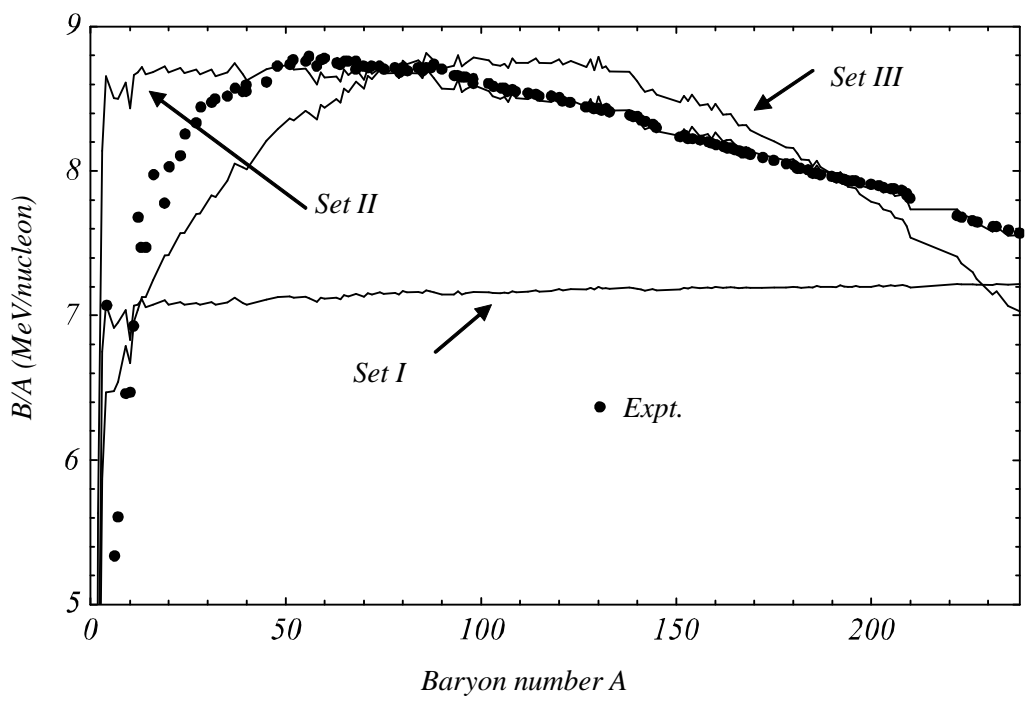

FIG. 1: Binding energy per nucleon $B / A$ as a function of the baryon number $A$ : The experimental data (black circles) are shown along with predicted values (solid lines) for parametrization of Set I $(\alpha=\beta=0)$, Set II (best fit for nuclear masses), and Set III (best fit for $B / A$ ), respectively.

Comparing Set II and Set III to the original Skyrme model with a pion mass term, we may identify

$$
F_{\pi}=4 \sqrt{\alpha}, \quad e^{2}=\frac{1}{32 \beta}
$$

and using (19) we find

$$
m_{\pi}=1.0621 \frac{\mu}{\sqrt{\alpha}}
$$

These quantities, $F_{\pi}, e^{2}$ and $m_{\pi}$ take values (see Table I) which are orders of magnitude away for those obtained for the Skyrme model but this is expected since we have assumed from the start that $\alpha$ and $\beta$ are relatively small.

We find also that the Skyrme term has the wrong sign so it would destabilize the soliton against shrinking if it was not for the contribution of order six in derivatives which ensures stability against scale transformations. Indeed the term of order six was even introduced at one point to resolve some problems with this sign [12]. In principle however, a negative coefficient for the Skyrme term could become problematic since the energy may no longer be bounded from below. One can argue that for our set of parameters, the relative weight of the $\mathcal{L}_{4}$ piece with respect to that of $\mathcal{L}_{0}$ or $\mathcal{L}_{6}$ is so small, i.e. approximately $\beta \nu^{1 / 3} / \lambda \mu \sim-10^{-6}$, and is at least partially canceled by that of the nonlinear $\sigma$ term $\mathcal{L}_{2}$ so that the energy would remain bounded from below. To substantiate this point on the relative contribution of each term, it is useful at this point to invoke some relevant links noticed by Manton 27] between an effective $S U(2)$ scalar Lagrangian and the strain tensor in the theory of elasticity. As in nonlinear elasticity theory, the energy density of a Skyrme field depends on the local stretching associated with the map $U: R^{3} \mapsto S^{3}$. This is related to the strain tensor at a point in $R^{3}$ which is defined as

$$
\begin{aligned}
M_{i j} & =\partial_{i} \Phi \partial_{j} \Phi \quad \text { where } \Phi=\left(\sigma, \pi^{z}, \pi^{x}, \pi^{y}\right) \\
& =-\frac{1}{4} \operatorname{Tr}\left[\left\{L_{i}, L_{j}\right\}\right]
\end{aligned}
$$

where $i, j$ refers to the Cartesian space coordinates. $M_{i j}$ is a $3 \times 3$ symmetric matrix with three positive eigenvalues $\lambda_{1}^{2}, \lambda_{2}^{2}$, and $\lambda_{3}^{2}$. Three fundamental invariants emerges from $M_{i j}$ in this simple geometrical interpretation due to Manton. They correspond to the Lagrangians $\mathcal{L}_{2}, \mathcal{L}_{4}$, and $\mathcal{L}_{6}$ and lead to the following energy densities, respectively:

$$
\begin{aligned}
& \mathcal{E}_{1}=\alpha\left(\lambda_{1}^{2}+\lambda_{2}^{2}+\lambda_{3}^{2}\right) \\
& \mathcal{E}_{2}=-|\beta|\left(\lambda_{1}^{2} \lambda_{2}^{2}+\lambda_{2}^{2} \lambda_{3}^{2}+\lambda_{1}^{2} \lambda_{3}^{2}\right) \\
& \mathcal{E}_{3}=3 \gamma \lambda_{1}^{2} \lambda_{2}^{2} \lambda_{3}^{2}
\end{aligned}
$$


where we wrote for simplicity $\gamma=\frac{3}{2} \frac{\lambda^{2}}{16^{2}}$, and to the baryon density

$$
\mathcal{B}^{0}=\frac{1}{2 \pi^{2}} \sqrt{\lambda_{1}^{2} \lambda_{2}^{2} \lambda_{3}^{2}}
$$

Assuming without loss of generality that $\lambda_{1}^{2} \geq \lambda_{2}^{2} \geq \lambda_{3}^{2}$, we find

$$
\mathcal{E}_{1} \geq 3 \alpha \lambda_{3}^{2}, \quad \mathcal{E}_{2} \geq-3|\beta| \lambda_{1}^{2} \lambda_{2}^{2}, \quad \mathcal{E}_{3} \geq 3 \gamma \lambda_{1}^{2} \lambda_{2}^{2} \lambda_{3}^{2}
$$

for a total energy density

$$
\mathcal{E} \geq \mu^{2} V+\frac{\alpha|\beta|}{\gamma}+3\left(\lambda_{3}^{2}-\frac{|\beta|}{\gamma}\right)\left(\alpha+\gamma \lambda_{1}^{2} \lambda_{2}^{2}\right) \geq \mu^{2} V-3|\beta|\left(\lambda_{1}^{2} \lambda_{2}^{2}\right)
$$

So, negative energy density contributions would come from regions where $\lambda_{3}^{2}<\frac{|\beta|}{\gamma} \sim-10^{-6}$, in other words, where the baryon density $\mathcal{B}^{0}$ in (60) is very small. Even for $\lambda_{3}^{2}=0$ and $\alpha$ negligibly small, the energy density should be dominated by the potential term $\mu^{2} V$. If we consider the integrated energy density subject to the condition that the total baryon number $A$ is a positive integer, then we expect the energy to be bounded from below for our set of parameters.

Clearly for our axial solution, the $\mathcal{L}_{2}$ and $\mathcal{L}_{4}$ pieces of the Lagrangian do not play the same significant role in the stabilization of the soliton as they do in the case of the Skyrme model. The properties of the soliton are almost completely determined by the values of $\mu$ and $\lambda$ so $F_{\pi}$ and $m_{\pi}$ may not be so closely related to the nucleon mass scale as for the original Skyrme model. Perhaps the explanation for such a departure is that the parameters of the model are merely bare parameters and they could differ significantly from their renormalized physical values. In other words, we may have to consider two quite different sets of parameters: a first one, relevant to the perturbative regime for pion physics where $F_{\pi}$ and $m_{\pi}$ are close to their experimental value and a second one, that applies to the nonperturbative regime in the case of soliton. Unfortunately, one of the successes of the original Skyrme model is that it established a link between pion physics with realistic values for $F_{\pi}$ and $m_{\pi}$ and baryon masses. Such a link here is more obscure.

On the other hand, the model in (1) (in the regime where $\alpha$ and $\beta$ are small) improves the prediction with regard to the properties of the nuclei of nuclear masses. Let us look more closely at the results presented in Fig. 1 These are in the form of the ratio of the binding energy per nucleon $(B / A)$ as a function of the baryon (or atomic) number $A$. The experimental data (black circles) are shown along with predicted value (solid lines) for parametrization of Set I, Set II and Set III. Set I is the least accurate when it comes to reproducing the experimental data, especially in the heavy nuclei sector. Yet, the agreement remains within a $0.4 \%$ of the experimental masses which is much better than with the original Skyrme model. Moreover, since the ratio $B / A$ depends on the difference between the mass of a nucleus and that of its constituents, it is sensitive to small variation of the nuclear masses so the results for $B / A$ may be considered as rather good. The second fit (Set II), which is optimized for nuclear masses, overestimates the binding energies of the lightest nuclei while it reproduces almost exactly the remaining experimental values ( $A \gtrsim 40$ ). Finally, the least square fit based on $B / A$ (Set III) is the best fit overall but in order to provide a better representation for light nuclei, it abdicates some of the accuracy found in Set II for $A \gtrsim 40$.

This apparent dichotomy between light and heavy nuclei may be partly attributed to the (iso)rotational contribution to the mass. The size of nuclei grows as $A^{\frac{1}{3}}$ and their moments of inertia increase accordingly. Also, the spin of the most abundant isotopes remains small while isospin can have relatively large values due to the growing disequilibrium between the number of proton and the number of neutron in heavy nuclei. Our numerical calculations reveal that the total effect leads to a (iso)rotational energy $E_{\mathrm{rot}}<1 \mathrm{MeV}$ for $A>10$ for all sets of parameters considered and its contribution to $B / A$ decreases rapidly as $A$ increases. On the contrary for $A<10$ the rotational energy is responsible for a larger part of the binding energy which means that $B / A$ should be sensitive to the way the rotational energy is computed. So clearly, the shape of the baryon density will have some bearing on the predictions for the small $A$ sector.

Since part of this work is to propose a model with nonshell configuration, it is relevant to compare our result with a similar analysis 21] which involves a typical shell-like configuration. For this purpose we repeated our calculation omitting the Coulomb and isospin breaking term. It turns out that both models are equally successful at reproducing data. Minimizing the square root of the mean squared deviation of $B / A$ from its experimental value gives almost identical results for both models, $\sigma=0.50 \mathrm{MeV}$ per nucleon, despite generating completely different baryon and energy configurations. In fact, the absence of a variation in $\sigma$ signals somehow the equal inability for both models to provide an accurate description of light and heavy nuclei sectors at the same time. One could improve the agreement by fitting separately the parameters $\mu, \alpha, \beta$, and $\lambda$ in the two sectors $A>40$ and $A<40$. But this would means introducing an arbitrary baryon number dependence on the parameter which could only be justified by introducing some kind of dynamical effect on $\mu, \alpha, \beta$, and $\lambda$. 


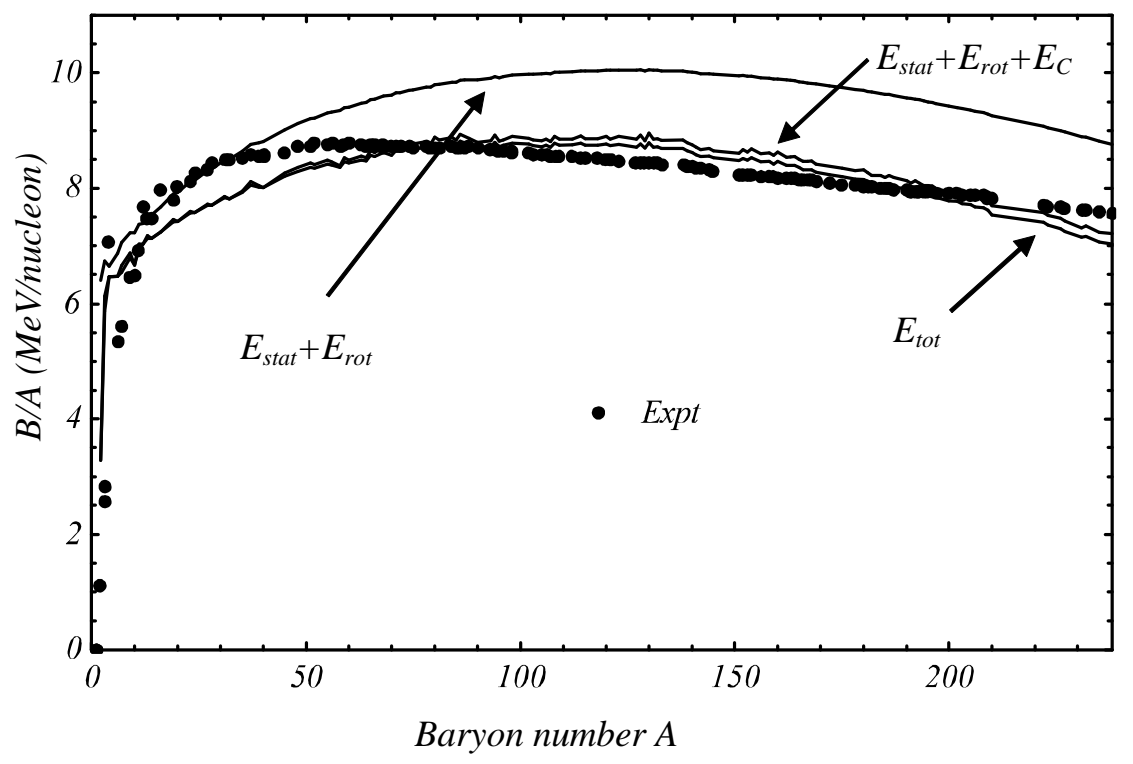

FIG. 2: Contributions of the Coulomb and isospin effect to the binding energy per nucleon $B / A$ as a function of the baryon number $A$ for Set III: The experimental data (black circles) are shown along with contributions due to $E_{\text {stat }}+E_{\text {rot }}, E_{\text {stat }}+$ $E_{\mathrm{rot}}+E_{C}$, and $E_{\mathrm{tot}}=E_{\mathrm{stat}}+E_{\mathrm{rot}}+E_{C}+E_{I}$ (solid lines).

The second motivation for this work regards this addition of the Coulomb and isospin breaking effect into the nuclear masses. These are often neglected in the context of the Skyrme model although they must inevitably be taken into account for a complete description of the nucleus. The Coulomb energy and isospin breaking term represent small corrections to the nuclear mass (of the order of $0.1 \%$ and $0.01 \%$, respectively) however our results show that the Coulomb effect is much more significant in the calculation of the binding energy. The change in $B / A$ is depicted in Fig. 2 for Set III in the separation between the red and blue lines (with and without Coulomb term, respectively). The effect increases almost linearly with the baryon number up to approximately $2 \mathrm{MeV}$ per nucleon for the heaviest nuclei. It represents roughly half the Coulomb effect estimated in the Liquid Drop model $(B / A)_{\text {Coulomb }}=-a_{C} Z(Z-1) A^{-4 / 3}$ where the value of $a_{C}=0.691 \mathrm{MeV} /$ nucleon. On the other hand, the isospin breaking contribution due to $E_{I}$ remains very small. Despite the magnitude of these corrections, it turns out that the optimization of the model parameters only yield, a slight improvement of the overall agreement with $\sigma=0.48 \mathrm{MeV}$ per nucleon.

To summarize, we have proposed a 4-terms model as a generalization of the Skyrme model. By choosing an appropriate form for the potential $V$, we allowed for near-BPS solitons with nonshell configurations for the baryon density in order to achieve a more realistic description of nuclei as opposed to the more complex configurations found in most extensions of the Skyrme model (e.g. $A=2$ toroidal, $A=3$ tetrahedral, $A=4$ cubic,...). Moreover, we introduced additional contributions to the mass of the nuclei coming from the Coulomb energy and an isospin breaking term. Fitting the model parameters, we find a remarkable agreement for the binding energy per nucleon $B / A$ with respect to experimental data. These results suggest that nuclei could be considered as near-BPS Skyrmions. On the other hand, there remain some caveats. First, the Skyrme model provides a simultaneous description for perturbative pion interactions and nonperturbative baryons physics with single realistic values for $F_{\pi}$ and $m_{\pi}$ and baryon masses. The connection between the two sectors here seems to be much more intricate. Also, a much better agreement could be reached if one could construct a solution that would describe equally well the light and heavy nuclei. Finally, one would like ultimately to reproduce the observed structure of the nucleus, i.e. a roughly constant baryon density becoming diffuse at the nuclear surface which is characterized by a skin thickness parameter. A more appropriate choice of potential may be instrumental in achieving some of these goals.

This work was supported by the National Science and Engineering Research Council of Canada.

[1] T.H.R. Skyrme, Proc. Roy. Soc. Lond. A, 260:127-138, 1961; T.H.R. Skyrme, Proc. Roy. Soc. Lond. A, 247:260-278, 1961; T.H.R. Skyrme, Nucl. Phys. 31:556-569, 1962; T.H.R. Skyrme, Proc. Roy. Soc. Lond. A, 247:260-278, 1958. 
[2] G. t Hooft, Nuclear Physics B, 72:461-473, 1974.

[3] E. Witten, Nuclear Physics B, 160:57-115, 1979.

[4] G. S. Adkins,C. R. Nappi and E. Witten, Nucl.Phys.B228:552,1983.

[5] L. Marleau, Phys. Rev. D, 43:885-890, 1991.

[6] E. Bonenfant and L. Marleau, Phys. Rev. D, 80:114018, 2009.

[7] V. B. Kopeliovich, B. Piette and W. J. Zakrzewski, Phys. Rev. D, 73:014006, 2006.

[8] P. Sutcliffe, Phys. Rev. D, 79:085014, 2009.

[9] G. S. Adkins and C. R. Nappi, Phys. Lett. B, 137:251-256, 1984.

[10] P. Sutcliffe, JHEP 1008:019, 2010.

[11] C. Adam, J. Sanchez-Guillen and A. Wereszczynski, Phys.Lett.B691:105-110,2010.

[12] A. Jackson, A. D. Jackson, A. S. Goldhaber, G. S. Brown, and L. C. Castillo, Phys. Lett. 154B, 101,1985

[13] G. S. Adkins and C. R. Nappi, Nucl. Phys. B, 233:109-115, 1984.

[14] M. Durgut and N. K. Pak, Phys. Lett. B 159, 357 (1985) [Erratum-ibid. 162B, 405 (1985)].

[15] G. Kalbermann, Phys. Rev. C 34, 2341 (1986).

[16] A. Ebrahim and M. Savci, Phys. Lett. B 189, 343 (1987).

[17] P. Jain, R. Johnson, N. W. Park, J. Schechter and H. Weigel, Phys. Rev. D 40, 855 (1989).

[18] H. Weigel, J. Schechter and N. W. Park, In *Syracuse 1989, Proceedings, 11th Annual Montreal-Rochester- SyracuseToronto Meeting* 110-130. (see Conference Index)

[19] E. Rathske, Z. Phys. A 331, 499 (1988).

[20] U. -G. Meissner, A. M. Rakhimov, A. Wirzba and U. T. Yakhshiev, EPJ Web Conf. 3, 06008 (2010) arXiv:0912.5170 [nucl-th]].

[21] E. Bonenfant and L. Marleau, Phys.Rev. D, 82:054023, 2010.

[22] C. Houghton and S. Magee, Physics Letters B, 632:593-596, 2006.

[23] J.-P. Longpré and L. Marleau, Phys. Rev. D, 71:095006, 2005.

[24] C.J. Houghton, N.S. Manton and P.M. Sutcliffe, Nucl. Phys. B510:507, 1998.

[25] B.C. Carlson and G.L. Morley, Amer J. Phys. 31:209, 1963.

[26] See for example K. S. Krane, Introductory Nuclear Physics, John Wiley and sons, p. 67, 1987 or more recent G.Audi, A.H.Wapstra and C.Thibault, Nuclear Physics A729: 337-676 (2003).

[27] N.S. Manton, Commun. Math. Phys. 111, 469 (1987). 